\title{
Artigo
}

\section{Pesquisa qualitativa em debate: entre especificidades e "novas" concepções nos estudos comparativos ${ }^{1}$}

\author{
Qualitative research in debate: between specificities \\ and "new" conceptions in comparative studies
}

\section{Patricia Benedita Aparecida Braga}

Doutoranda do Programa de Pós-graduação em Ciência Política, Universidade Federal de São Carlos (UFSCar), São Carlos, SP, Brasil bragapba@gmail.com

\begin{abstract}
Resumo: Na Ciência Política, há uma vasta área de reflexões a respeito dos aspectos teóricos e metodológicos empreendidos em pesquisas científicas. Dentre essas discussões, destacam-se os acalorados debates entre os métodos quantitativos e qualitativos, por meio da demarcação de fronteiras analíticas. No campo da Política Comparada, esse limite fronteiriço entre as pesquisas qualitativas e quantitativas também se faz presente. Nesse contexto reflexivo e metodológico, baseado no que foi denominado ao longo da década de 1990 de "nova metodologia qualitativa", o presente artigo defende que é possível empreender abordagens quantitativas e qualitativas em uma única pesquisa, embora haja princípios específicos, nas duas perspectivas, que devem ser respeitados. Por conseguinte, inserido nas reflexões realizadas pela "nova metodologia qualitativa" em relação à condução analítica dos estudos de casos comparados qualitativamente este artigo objetiva divulgar a perspectiva analítica de Sheila Jasanoff sobre a feitura de análises em política comparada, assim como uma reflexão sobre possíveis diálogos e limites analíticos dessa perspectiva.
\end{abstract}

Palavras-chave: Nova metodologia qualitativa; Política comparada; Coprodução.

\begin{abstract}
In Political Science there is a wide field for reflections about the theoretical and methodological aspects undertaken in scientific researches. Among these discussions, there is the heated debate between the quantitative and the qualitative methods, through
\end{abstract}

1 Este artigo foi premiado como um dos melhores artigos no II Workshop de Metodologia em Ciência Política UNICAMP/UFSCar, que ocorreu entre os dias 21 de novembro a 2 de dezembro de 2016. 
demarcation of analytic frontiers. In the Comparative Politic field this bordering limit, between the quantitative and qualitative research, is also present. In this reflexive and methodological context, based in what was called along the 1990's the "new qualitative methodology", the present article seeks to argue that is possible to undertake some approaches of qualitative and quantitative in one single research, but there is specific principles in these two perspectives that should be respected. Consequently, inserted in the reflections accomplished by the "new qualitative methodology", in regard of the analytic conduction of studies in qualitative comparative cases, the aim is proclaim an reflection of Sheila Jasanoff's analytical perspective about the making of analysis in comparative politics, as an reflection about the possible dialogues and analytic limits of this perspective.

Keywords: "New quantitative methodology"; Comparative politics; Coproduction.

\section{Introdução}

É lugar comum nas discussões contemporâneas sobre metodologia da Ciência Política o debate sobre as possibilidades ou não da correlação entre métodos qualitativos e quantitativos, sendo um bom exemplo dessa discussão, no campo da Política Comparada, a obra de Robert Putnam, Making Democracy Work (PUTNAM, 1993). Essa obra mostra que, se por um lado existe o compromisso e o domínio de uso dos métodos quantitativos e da análise estatística, considerados profícuos na produção de inferências causais, há, por outro lado, um amplo debate sobre a produção de inferência causal em pesquisas qualitativas small-n.

Considera-se pouco vantajoso considerar os desenhos de pesquisas, sejam eles de abordagem quantitativa ou qualitativa, de modo antagônico, ou seja, como fronteiras analíticas, que precisam ser reforçadas e criticadas. As reflexões seriam mais frutíferas se as abordagens qualitativas e quantitativas fossem consideradas pressupostos analíticos que poderiam auxiliar-se na compreensão de objetos em determinados desenhos de pesquisas, respeitando, obviamente, seus pressupostos ontológicos e epistemológicos.

Logo, refletir sobre a integração ou a demarcação entre as perspectivas qualitativas e quantitativas, a partir do respeito aos pressupostos específicos dessas duas perspectivas analíticas, não significa demarcar fronteiras em termos metodológicos, mas refletir teoricamente as possibilidades e os limites desse diálogo e construir possibilidades outras de desenhos de pesquisa dentro do que é denominado política comparada.

Diferentemente do que argumentam King, Keohane e Verba (1994) a respeito da lógica das pesquisas qualitativas que deveriam utilizar os preceitos das análises quantitativas, considera-se o argumento de Mahoney (2010), de que há traços em desenhos de pesquisa qualitativas e quantitativas que são específicos. 
Portanto, o presente artigo, dentro das discussões metodológicas de Política Comparada qualitativa, tem por objetivo não só apresentar a perspectiva analítica de Sheila Jasanoff, mas também problematizar possíveis diálogos e limites dessa vertente - inflexões. Com o intuito de cumprir tal meta, o artigo estrutura-se do seguinte modo: 1) debate entre as perspectivas analíticas qualitativas e quantitativas em política comparada e as três condições essenciais nos desenhos de pesquisas em política comparada qualitativa; 2) política comparada e estudo de casos comparados qualitativos; e, por fim, 4) a perspectiva de Sheila Jasanoff sobre a análise de política comparada - limites e diálogos.

\section{O debate analítico em política comparada de análises quantitativas e qualitativas}

A fim de testar e descobrir, de modo indutivo, novas hipóteses, assim como construir teorias, o método comparativo é um dos principais métodos na ciência política contemporânea (COLLIER, 1993). Distinto do método experimental e estatístico, essa análise pode trabalhar com variáveis múltiplas e com um pequeno número de casos, sem que isso represente um problema, como expôs Lijphart na obra Comparative Politics and the Comparative Method, de 1971.

Ao longo da década de 1990, para legitimar a escolha por análises voltadas a poucos casos e desmistificar o problema da existência de muitas variáveis em cada caso (many variables, small-n) (COLLIER, 1993), expandiu-se o número de debates na academia, entre os metodólogos, a respeito de abordagens analíticas baseadas no método comparativo. Essas análises direcionam o olhar a um número pequeno de casos e, sob certos preceitos, eram tão boas em termos objetivos quanto as análises estatísticas dos métodos quantitativos, mas com concepções teóricas/metodológicas distintas.

De acordo com Collier (1993), um dos objetivos das análises comparativas deveria ser o de avaliar as explicações concorrentes, com três razões: 1) exame sistemático da covariância entre os casos, com o intuito de efetuar análises causais; 2) análise de um pequeno número de casos deveria não só ofertar teste de hipóteses, como também construir teorias, pois a aplicação de um conceito para um número expressivo de casos poderia gerar generalizações e distinções entre as unidades comparadas; e, por fim, 3) em razão da valoração contextual e da análise interpretativa no método comparado, o número de casos não deveria ser expandido (COLLIER, 1993). 
De modo distinto e duas décadas antes, Przeworski e Teune (1970) afirmaram que as análises não necessariamente deveriam estar vinculadas a casos comparáveis e que a escolha de casos distintos seria mais frutífera, se essas análises ocorressem por meio de ferramentas específicas, como o process tracing 2 , o pattern matching ${ }^{3}$ e o with-in case analysis ${ }^{4}$, como também as variáveis explicativas não deveriam ser reduzidas, pois a formação de conceitos e tipologias seria substancial na análise comparada (GEDDES, 1991).

Logo, se, por um lado, as análises qualitativas buscam realizar análise por meio de estudo de casos, análises small-n, construção de conceitos, etnografia, método histórico-comparativo e interpretação, por outro lado, as análises quantitativas fazem análises sobre um número alto de casos por meio de análises de regressão e técnicas de inferência causal, fundamentando-se em medidas numéricas e em generalizações das descrições para a realização do teste de hipóteses (BRADY; COLLIER; SEAWRIGHT, 2004).

Portanto, ao debater metodologicamente sobre a análise comparada, também se debate a existência ou não de diferenças essenciais entre o método quantitativo e o qualitativo. Esse debate ocorre porque, enquanto a análise quantitativa faz uso de experimentos de controle e estima o efeito médio das variáveis independentes em uma amostra, assim como examina os efeitos das causas, a análise qualitativa observa diversos caminhos causais que levam a resultados singulares em cada caso ou em alguns casos (PASQUARELLI, 2014).

Os efeitos médios, nas análises quantitativas, só podem ser estimados por meio do controle das variáveis consideradas relevantes ao estudo, pois as causas afetam os valores de um resultado em uma análise de amostragem ampla. Já no método qualitativo, os casos são selecionados pela variável dependente e representam o escopo da teoria, cuja expansão acaba por gerar modificações no próprio modelo teórico em uso; portanto, alguns casos são identificados como mais significativos que outros.

20 process-tracing é uma ferramenta utilizada para analisar processos de mudança ao longo de um período histórico (não linear), evidenciando mecanismos e sequências causais em contextos específicos (COLLIER; SEAWRIGHT, 2004).

3 Já o pattern matching, também usado em pesquisas qualitativas, tem como função procurar e encontrar padrões de correspondência entre a teoria utilizada e os dados do contexto de análise (TROCHIM, 1989).

4 O with-in case analysis examina um ou poucos casos. De acordo com Seawright e Collier (2004), essa análise pode ser realizada de duas maneiras. A primeira, dentro da tradição qualitativa, buscando demonstrar internamente a evidência de padrões de causalidade relacionados a inferências externas associadas ao caso particular. De outro modo, podem-se também coletar observações da variável dependente e das variáveis independentes para subunidades múltiplas do caso original. 
Na análise quantitativa, há um amplo escopo de casos e as generalizações são produzidas por meio da exclusão de variáveis consideradas menos importantes e outras variáveis independentes são selecionadas de modo aleatório. Logo, a atenção está direcionada a sua própria operacionalização e ao uso de indicadores, cujos erros de mensuração são selecionados com a inclusão de novos indicadores (MAHONEY; GOERTZ, 2006).

Na análise qualitativa, a busca é por solucionar problemas minuciosos e explicar resultados específicos, com base na coleta de dados detalhados, nas semelhanças de casos e no conhecimento de princípios causais. Portanto, é um teste de teoria, pois um dado pode afetar as evidências encontradas e o erro pode levar à revisão conceitual (MAHONEY; GOERTZ, 2006).

Contudo, na obra de King, Keohane e Verba (1994), essas distinções entre as análises quantitativas e qualitativas não são autodeclaradas como antagônicas, mas "integradas", "associadas", pois ambos os desenhos de pesquisa são baseados em uma mesma lógica de inferência, a descritiva (observação do mundo para apreender fatos alheios, generalizando uma amostra de um universo de caso e criando conceitos sobre a estrutura social) e a exploratória (observação dos efeitos dos dados, comparação de algo que ocorreu com algo que não ocorreu), cujos resultados devem ser julgados a partir do método empregado.

Tem-se, pois, que a produção da inferência deve ser central no estilo de pesquisa, e isso deve ser realizado por meio de regressão. Portanto, as pesquisas qualitativas deveriam orientar-se por regras e procedimentos das análises quantitativas, já que as análises qualitativas possuem viés de seleção (selection bias) e generalização, colocando em restrições a variável dependente e, por sua vez, não produzindo inferências causais. Além disso, quanto maior for o número de variáveis em relação ao número de casos, maior será a indeterminação da generalização, gerando não confiabilidade nas análises (PASQUARELLI, 2014).

Esse entendimento de King, Keohane e Verba (1994) sobre a produção de inferências nos desenhos de pesquisas, assim como a eloquência na defesa de um método sobre o outro, recebeu críticas, principalmente com o advento das reflexões do que se denominou de "nova metodologia qualitativa", para a qual a pesquisa qualitativa possui condições essenciais na condução de pesquisas. Ou seja, há uma inadequação entre pesquisas 
que utilizam os pressupostos/ferramentas do método quantitativo em pesquisas small-n e históricas, como o uso da inferência causal, pois há diferenças essenciais, como a natureza da explicação, a concepção de causalidade e os métodos utilizados para o teste de teorias (MAHONEY; TERRIE, 2008).

Nesse sentido, problematizada por Mahoney (2010) e outros metodólogos e tipificada por Rezende (2011a), a "nova metodologia qualitativa" afirma que há três condições essenciais da pesquisa qualitativa: a) as relações entre causas e efeitos; b) a especificidade do fenômeno político; e, c) a interferência de agências, instituições e mecanismos causais na explicação política, além do fato de que os estudos quantitativos, por não realizarem adequadamente a produção de inferências causais, não devem ser o parâmetro por excelência nas pesquisas comparadas em ciência política.

\section{Causas e efeitos}

Enquanto o objetivo da análise estatística é produzir explicações sobre os efeitos das causas (effects-of-causes), os efeitos médios da causação e o emprego de técnicas de análise de regressão para o teste de hipótese, o método histórico-comparado busca compreender as causas que geram efeitos (cause-of-effects), com o intuito de produzir explicações, juntamente com uma lógica de condições essenciais para a análise dos processos de causação. Para tanto, usam-se o process-tracing para testes de teoria, além do uso da lógica confractual (e se), das técnicas de fuzzy-set e das análises path dependency (REZENDE, 2011a).

A diferença reside no modo como as perspectivas qualitativas constroem suas perguntas e, por conseguinte, geram suas explicações. Em pesquisas comparadas, usualmente desenvolvidas a partir de desenhos small-n, os cientistas políticos buscam compreender como um conjunto de causas (ou variáveis independentes), postas pela teoria que o pesquisador utiliza, gera o comportamento ou o efeito sobre a variável dependente. E, obviamente, como o contexto e as condições específicas de produção do fenômeno a ser estudado incidem sobre a produção da causalidade.

Se os aspectos contextuais incidem sobre a causalidade, um dos desafios é atentar para o viés de seleção dos casos e para a não generalização da análise por meio de critérios metodológicos. E isso ocorre pela concepção de que as teorias e os conceitos não são descontextualizados, como supõe a pesquisa comparada quantitativa, e que a heterogeneidade causal se estabelece na relação entre teorias e a população de casos. 
Ou seja, a partir do tipo de explicação que se pretenda, cada pesquisador realiza recortes em relação à heterogeneidade causal, sendo casos típicos as pesquisas embasadas em desenhos de pesquisa do tipo small-n e/ou estudos de caso, pois o contrário disso pode acarretar exclusão de fatores causais, assim como problemas na mensuração de variáveis de cada teoria.

Já nas pesquisas que objetivam realizar análise estatística, a inclusão de novos casos não é problemática durante o processo de análise, haja vista que os erros de mensuração podem ser modelados, pois o pressuposto da homogeneidade causal permite a construção de generalizações a partir da produção da estimação de efeitos médios e a apresentação de variáveis independentes.

\section{A singularidade dos fenômenos políticos}

Outra condição essencial da pesquisa qualitativa é que os fenômenos políticos não possuem, em sua natureza, condições, leis e regularidades que possam ser observadas repetidamente, o que dificulta a produção de inferências válidas e confiáveis ${ }^{5}$. Nas análises qualitativas, portanto, a partir da especificidade de seus métodos e desenhos de pesquisa, busca-se entendimento de um alto número de variabilidade das causas, assim como a conexão dessas causas com os múltiplos caminhos existentes entre causas e efeitos.

O intuito é, pois, procurar a variação das causas ou como elas se associam e produzem determinados efeitos, porque causas iguais podem produzir efeitos diversos, e mecanismos causais são invariantes. Portanto, para além de serem utilizados nos testes de hipóteses, os dados empíricos servem para a construção de entendimentos sobre os processos causais que ofertam sentido às variáveis estudadas a partir de uma teoria.

A conexão entre teorias e dados empíricos não é utilizada com frequência no teste de hipóteses ou na relação entre duas variáveis $X$ e $Y$, pois usualmente essa conexão serve para produzir conceitos, reformular ou expandir teorias ou para analisar maneiras com que as variáveis estão articuladas em contextos específicos.

Freedman (2008) realiza a distinção de dois tipos de pesquisa científica. Um, direcionado à análise estatística de dados e teste formal de hipóteses, direciona seu olhar para a formulação de hipótese e confronto com a realidade empírica, expondo a

5 Um dos exemplos disso é a eleição americana de 2016 para a presidência, na qual todas as pesquisas quantitativas, contestadas após a vitória do candidato republicano Donald Trump, previam a vitória da candidata democrata Hillary Clinton 
importância de evidências relacionadas a processos causais (causal process observation) em relação à produção de inferências causais (BRADY; COLLIER; SEAWRIGHT, 2004), cujo intuito é adotar relevância universal ou a invariância das causas.

Outro tipo de pesquisa destacado por Freedman (2008), voltado para o modelo qualitativo, defende que é necessário não apenas observar os mecanismos causais e as variáveis custosas à explicação de fenômenos políticos analisados, mas também observar o contexto e a complexidade como elementos causais pertinentes à produção de um determinado fenômeno, principalmente em pesquisas de estudo de caso.

Nesse sentido, é necessário atentar para o descompasso existente entre teorias e realidade empírica, pois o fundamento de uma parte ainda expressiva das teorias em política comparada tem por natureza a ideia de um mundo previsível, enquanto a não regularidade é a condição máxima da realidade, alimentando o descompasso entre as teorias escolhidas previamente e os eventos raros (black swans) que muitas vezes se tornam constâncias (BLYTH, 2006).

Outro ponto de distinção está, de um lado, na elaboração de um modelo causal com hipóteses que virão a ser testadas a partir de evidências (casos), denominado modelo frequentista, e, de outro lado, o conhecimento prévio sobre a realidade estudada e sobre os casos, como possíveis pressupostos de expansão ou refinamento de teorias e modelos existentes, identificado como lógica bayesiana (REZENDE, 2011a). O que leva à confirmação de que modelos causais são heterogêneos e singulares na ciência política, em razão da presença de contrafactualidades e múltiplas causas.

Portanto, sendo a realidade repleta de heterogeneidade e de contrafactuais (RAGIN, 1987; GOERTZ; STARR, 2002), assim como de variações dos padrões causais, a complexidade poderia ser apreendida por meio de estudos de caso, classificados como positivos e/ou negativos (MAHONEY; GOERTZ, 2004), com o intuito de compreender teoricamente os fatores causais que geram o comportamento da variável dependente.

Isso ocorreria a partir de determinados conceitos, como momentos críticos, path dependency, causação múltipla e equifinalidade nos estudos típicos de análise histórico-comparada que buscam ressaltar o problema das singularidades na análise política, mas que alertam para o fato de que a elevação do número de observação não necessariamente gera maior entendimento dos fenômenos políticos. Assim, para que haja um entendimento mais denso dos fenômenos político, é necessário adentrar os 
casos, assim como comparar a variabilidade causal entre esses, e, por conseguinte, talvez produzir teorias mais completas sobre a realidade (REZENDE, 2011a, b).

Nas pesquisas qualitativas, portanto, as condições necessárias são decisivas para possivelmente entender os padrões variáveis de causação, enquanto, nas pesquisas quantitativas, há a utilização do princípio de correlação para a compreensão de um dado fenômeno por meio de um conjunto de causas (GOERTZ; STARR, 2003).

As explicações causais de fenômenos políticos estão ancoradas essencialmente na maneira como agentes (agência), regras do jogo (instituições formais e informais) e mecanismos causais geram o comportamento da variável dependente, e nisso se constitui a terceira diferença essencial entre métodos qualitativos e quantitativos na política comparada.

\section{Agências, instituições e mecanismos causais}

Os desenhos de pesquisa devem priorizar amostras reduzidas, análises histórico-comparadas, instituições e a produção da ação e do comportamento político (REZENDE, 2011a) nos estudos qualitativos.

Os atores (agências) são dotados de racionalidade, construída pelos desejos e crenças que permeiam os atores sociais. Logo, a escolha dos agentes gera comportamento e ação política (PLOTT, 1991). Nesse sentido, as agências não se dão apenas no âmbito coletivo, mas também na forma individual, organizacional, humana e não humana, havendo, portanto, entrelaçamento entre níveis micro e macro da ação causal (COLEMAN, 1990).

Se a ação e o comportamento político podem produzir explicações sobre um dado fenômeno político, esses dois elementos não são cindidos de paradigmas analíticos, como os neo-institucionais e as teorias da escolha racional, que podem vir a produzir teorias na política comparada. As Narrativas Analíticas (BATES et al., 1998; LEVI, 2004) constituem um exemplo clássico dessa condição, cujo enfoque é a compreensão da mudança e variabilidade nas instituições políticas, a partir de uma combinação entre pesquisa histórico-comparada e o uso intensivo de estudos de casos com modelos formais advindos da escolha racional, que é uma combinação entre os modelos formais da teoria dos jogos (instituições compreendidas a partir de jogos e equilíbrios) e a imprevisibilidade contextual produzida historicamente. 
Tanto as teorias neo-institucionais como as teorias dos jogos frisam a importância da agência no entendimento de fenômenos políticos, pois, por meio das narrativas produzidas pelos agentes, em uma dinâmica causal historicamente contextual (LEVI, 2004), é possível produzir inferências causais.

Nas Narrativas Analíticas, a inferência causal é demonstrada por meio do process-tracing e dos mecanismos causais que apregoam como as instituições e as escolhas diversas dos diferentes agentes constituem os fenômenos políticos. Isso ocorre por meio de desenhos de pesquisa que focam na construção de uma análise comparada de caso, que tem por objetivo realizar observação do processo causal com o intuito de produzir inferências, mesmo que essa técnica (a busca de conexões causais entre as variáveis independentes e dependentes num dado modelo causal) possa gerar uma regressão ao infinito, como exposto por King, Keohane e Verba (1994).

Dependente do contexto, da história e das escolhas contingencias dos agentes, a Narrativa Analítica busca compreender a múltipla causação, por meio dos casos e de observações densas sobre os processos causais (BRADY; COLLIER; SEAWRIGHT, 2004).

Distinta das regressões efetuadas nas análises estatísticas, a lógica bayesiana, base da "nova metodologia qualitativa", destaca os mecanismos causais na produção de inferências na pesquisa comparada, mesmo que isso não seja uma regra absoluta, ou seja, a compreensão da causação está relacionada à adesão das observações em relação aos modelos causais e suas hipóteses.

Contudo, é necessário ter em mente que nem sempre haverá conexão entre teoria e dados empíricos, o que não necessariamente deve ser apreendido como algo negativo nas pesquisas, haja vista que a possibilidade de criação de modelos e interpretações "novas" é benéfica, uma vez que as crenças e as compreensões teóricas dos pesquisadores em relação à realidade podem se modificar, o que Merton (1967) denomina de incerteza.

Logo, se há condições essenciais específicas entre as análises qualitativas e quantitativas, fruto de desenhos de pesquisa específicos, que Rezende (2011a; 2011b) expõe didaticamente e com que o presente artigo concorda, um próximo passo é refletir sobre as especificidades das análises comparativas e dos estudos de casos, em pesquisas qualitativas, e verificar se esses desenhos podem ser compreendidos como métodos distintos ou não. 


\section{Estudo de caso comparado na "nova metodologia qualitativa"}

Se, por um lado, a política comparada foi desde sua origem considerada uma das formas de análise da ciência política, por outro lado, os estudos de caso até recentemente não possuíam esse status, pois eram considerados uma metodologia de segunda categoria, com limites na produção de teorias e na geração de proposições causais para o conhecimento.

Com o surgimento do que se denominou "nova metodologia qualitativa", porém, a ciência política contemporânea passou a produzir amplos debates, a partir da década de 1990, sobre os limites de seus métodos analíticos e possível validade dos estudos de caso. O status científico dessa reflexão está relacionado aos conceitos e concepções que os intelectuais possuem sobre ciência, metodologia e formas de pesquisa na ciência política comparada.

Neste artigo, parte-se da proposição de que os estudos de caso podem se beneficiar das reflexões em torno do método comparado e vice-versa, com validade, portanto, com status legítimo, dentre as diversas formas de análise que fundamentam os desenhos de pesquisa em ciência política. Nesse caso, não seria mais considerado de segunda categoria, pois o próprio termo é juízo de valor e não reflete as posições epistemológicas da maioria dos cientistas políticos.

Alguns autores afirmam que os estudos de caso derivam-se dos métodos comparados, outros expõem que ambos são métodos específicos, em decorrência de seus princípios distintos. E, neste artigo, parte-se do entendimento de que o método comparado e os estudos de caso são correlacionados e podem ser definidos pelo enfoque analítico e técnico sobre os objetos de pesquisa.

De acordo com Yanow, Schwartz-Shela e Freitas (2008), a conceituação de estudos de caso se dá em dois polos, um de explicação causal e outro interpretativo. O primeiro (dominante na metodologia qualitativa da ciência política comparada), de explicação causal, compreende os estudos de caso como um conjunto de métodos utilizados na pesquisa comparativa (multi-site) que busca produzir inferências causais e teste de hipóteses. Nesse caso, os desenhos de pesquisa são moldados com o intuito de compreender, com base em teorias e modelos causais, as semelhanças e as diferenças entre os casos, combinando técnicas qualitativas e quantitativas de observação da realidade e com um limite de até seis casos de comparação. 
Contudo, como Ragin (1987) expõe, mais do que focar nas relações causais, é necessário apreender as características e as condições que produzem o fenômeno estudado.

O segundo pólo, denominado interpretativo, enfatiza os aspectos relacionados ao significado, à interpretação e à reflexividade da ação social, sendo os desenhos de pesquisa do tipo single-site (caso único). Assim, os pesquisadores realizam um desenho de pesquisa que privilegia as narrativas, os aspectos etnográficos e outros elementos relacionados à descrição densa da realidade.

De acordo com Rezende (2011b), seja pela perspectiva da explicação causal seja pela interpretativa, a pesquisa de estudo de casos deve ser pensada como parte da pesquisa comparada, principalmente a de corte histórico-comparativo.

Lijphart (1971), um dos primeiro autores a dar ênfase à abordagem de estudo de caso de cunho histórico-comparativo, por meio da exposição das características básicas do método comparado, afirmava que havia distintos tipos de caso (os não teóricos; os interpretativos; os geradores de hipótese(s); os confirmativos ou os negatórios de teoria; e os desviantes) e argumentou que um único caso seria profícuo, porém não favorecia a construção de teorias de aplicação geral.

Dentre as tipificações de estudos de caso, os que geram mais hipótese(s), como os desviantes, são mais apropriados na construção de teorias, por meio da criação de novas hipóteses ou na estimulação e/ou refinamento das hipóteses existentes.

Essa abordagem analítica foi renovada ao longo dos anos por meio da sistematização dos procedimentos (COLLIER, 1993), por métodos de padrões de correspondência (CAMPBELL, 1975), pela criação de tipologias com o intuito de testar hipóteses (ECKSTEIN, 1975) e no exame dos acontecimentos ao longo do tempo de modo processual (GEORGE; MCKEOWN, 1985 apud PASQUARELLI, 2014).

Nesse sentido, os estudos de caso se fundamentam geralmente em três procedimentos típicos para o teste de teorias: as comparações controladas, os procedimentos congruentes e o process tracing, que se diferenciam entre si pelas observações realizadas.

As comparações controladas são aquelas observações entre os casos, com base em critérios específicos de seleção de $\operatorname{casos}^{6}$ (GERRING, 2007); já os procedimentos

6 De acordo com Gerring (2007) há uma tipologia composta por nove estratégias de seleção de casos para as pesquisas de estudos de caso em que os pesquisadores comparados devem se basear quando buscam respostas ao problema da seleção de casos na ciência política. Os casos são: típicos (representativos); diversos (heterogêneos); extremos; desviantes; exemplares; cruciais; pathway; similares; e, diferente. 
congruentes estão relacionados à busca por compreensão de padrões de congruência e incongruência entre valores observados e previstos pela teoria. Portanto, os desenhos de pesquisa são como observações comparadas entre vários casos ou intracasos ou análises do tipo process tracing que realizam observações geradas no interior dos casos (within case) (REZENDE, 2011b).

Além de profícuo em áreas onde o método estatístico é pouco efetivo em termos de resolução de problemas considerados complexos, o estudo de caso pode ter algumas vantagens, dentre elas, o de não realizar estreitamento conceitual (conceptual stretching), típico de análises quantitativas, gerando identificação e medição de indicadores que representam conceitos teóricos. Acrescente-se ainda que os estudos de caso possuem procedimentos para identificar novas hipóteses e variáveis por meio de casos desviantes, o que, por sua vez, pode gerar novas teorias que serão testadas por meio de evidências que não haviam recebido análise; trazem para as análises os fatores contextuais, permitindo o exame da função hipotética dos mecanismos causais e das variáveis intervenientes em cada caso; e abordam a complexidade causal (equifinalidade, os efeitos de interação complexa e a path dependency) (GEORGE; BENNETT, 2005).

Mas também há limites e críticas aos estudos de caso. Uma das críticas mais fortes é a que diz respeito à propensão, à seleção enviesada (selection bias), pois os casos não devem ser selecionados por meio da variável dependente (GEDDES, 1990). Essa seleção, contudo, como dito anteriormente, em contrapartida, ajuda a identificar variáveis descartáveis à análise.

Outra crítica está relacionada à dificuldade dos estudos de caso em estimar os efeitos causais das variáveis. Todavia, a causalidade pode ser retrucada por meio de uma análise do contexto. Há também crítica em relação à imprecisão das explicações rivais, pois, ao contrário das explicações estatísticas, o estudo de caso possui variáveis com distintas características que devem ser avaliadas, visto que, dentro de cada caso, existem diversos process tracing observáveis ao longo dos caminhos causais hipotéticos entre variáveis dependentes e independentes, o que, por sua vez, gera novas observações históricas e controle das explicações. Logo, a explicação histórica de cada caso deve conter a avaliação de cada passo da sequência de um determinado resultado (GEORGE; BENNETT, 2005).

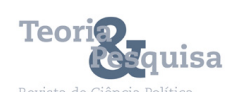


Outra crítica comum é a incapacidade que os estudos de caso têm de serem representativos, ou seja, seus resultados não se aplicam a populações mais amplas (MCKEOWN, 1994), ocorrendo o que os metodólogos denominam de trade-off, uma tensão entre o objetivo de alcançar alta validade interna e boas explicações históricas de casos particulares, em oposição à feitura de generalizações aplicadas a amostras amplas.

De acordo com George e Bennett (2005), o exame da história e dos processos causais forma a base da estrutura metodológica dos estudos de caso. Portanto, a análise do contexto de cada caso, a construção de uma narrativa cronológica e histórica, o estabelecimento dos valores das variáveis dependentes e independentes, a criação de explicações, tornam o process tracing uma ferramenta importante nesse tipo de pesquisa (PASQUARELLI, 2014).

O processs tracing identifica os processos causais intervenientes entre a variável independente e o resultado da variável dependente, rastreando processos que podem ter levado a um determinado resultado. E, juntamente com a equifinalidade, que considera caminhos alternativos, por meio dos quais os resultados podem ter ocorrido, o process tracing oferta um mapeamento ou caminhos causais distintos, mas consistentes com o resultado de um único caso ou mais. Essa ferramenta é, portanto, indispensável no teste e desenvolvimento de teoria, por meio da observância de atores, instituições, mas também por observações e explicações de um caso (GEORGE; BENNETT, 2005).

É necessário, no entanto, ter consciência de que a maioria dos fenômenos não são lineares - formas mais simples para a análise do processo causal - mas, ao contrário, são complexos, e a causalidade complexa origina-se de fluxos que convergem de distintas condições. É preciso atentar também para o fato de que, em casos compostos por uma sequência de eventos que impedem ou modificam trajetórias, denominados de path dependency, os fatores históricos influenciam os eventos no presente, o que, de acordo com Levi (1997), incidem sobre as tentativas de mudança.

Segundo Collier e Collier (1991) e Thelen (1999), a ideia principal do conceito de path dependency é a noção de conjuntura crítica, ou seja, um período de mudança que ocorre de modo diferente entre os países, a partir de rotas estabelecidas inicialmente, que demarcam a produção de legados distintos. Portanto, a conjuntura crítica pode ser uma transição política ou econômica vivenciada por países distintos, revolucionária ou institucional, cujo processo de mudança deixa um legado no qual as escolhas e as decisões ao longo do tempo tornam-se necessárias (FERNANDES, 2002). 
E, nesse sentido, apresenta-se no próximo tópico a perspectiva de Sheila Jasanoff sobre o que ela denomina de política comparada, mas que também poderia ser designado de estudo de casos comparados qualitativamente.

\section{O desenho de pesquisa comparada de Sheila Jasanoff}

Sheila Sem Jasanoff, professora do departamento de Ciência eTecnologia da Harvard Kennedy School, é autora de diversos livros e artigos nos quais reflete de modo geral as intrínsecas relações entre ciência, lei e política em diversos países, principalmente nos Estados Unidos, na Alemanha, na Inglaterra, na Coréia do Sul, na Índia, dentre outros, por meio de análises comparadas qualitativas.

Como Marques et al. (2012) expõe, todos os cientistas escrevem de onde estão e isso não é distinto no caso de Sheila Jasanoff. Mas o conhecimento que ela constrói, embora não possa mais ser compreendido como universal e neutro, pode ser traduzido, transladado, modificado, reinventado e situado localmente. Ou seja, as proposições que a autora realiza colocam em cena as justaposições de elementos que podem vir a configurar fatos ou instituições, até mesmo impérios. Contudo, essas ideias estão estritamente relacionadas com o que é feito com elas, como são utilizadas e modificadas.

É necessário, no entanto, expor que, por mais que a perspectiva de Jasanoff trabalhe com a ideia de desplatonização do mundo (RORTY, 1999) e apesar de seus diálogos com a perspectiva pós-colonial, principalmente com Said (1978), a respeito de como, nos estudos comparados, devem-se repensar continuamente a construção e a representação do 'Outro', assim como os essencialismos conceituais e explicativos (MAZZOCCHI, 2006), a autora não propõe uma perspectiva Sul versus Norte. Trata-se, de um (re) pensar metodológico a respeito do empreendimento de análises comparativas qualitativas que possuem o Estado-nação ainda como uma das unidades (casos) de comparação, mas em torno conceito de cultura política.

De acordo com essa perspectiva o campo da política (espaço dos valores e da ideologia) não estaria cindido do campo da ciência (conhecimento objetivo, empiricamente válido). A ciência e a política se constroem de modo relacional, por processos constantes, de mistura e separação, de conjunção e disjunção. 
As ações políticas e científicas estão relacionadas a ordenamentos legítimos existentes em específicos contextos sócio-históricos, portanto, estão relacionadas a compromissos políticos e a determinadas perspectivas analíticas, que se constroem cotidianamente, tanto em termos nacionais, como em termos inter-nacionais.

Nesse contexto, a democracia moderna, que tem como um dos seus princípios culturais a valorização da racionalidade científica e do pensamento objetivamente produzido, deve ser observada pela lente da coprodução, pois ambos os campos se constroem performaticamente e materialmente, com distintos modos de ordenamento que se retroalimentam (LAW, 1994). Contudo, essas construções não são isentas de lógicas assimétricas de poder, tanto em níveis micro, como entre indivíduos, como em níveis macro, entre nações, organizações, agências, dentre outros.

Se a ciência e a política não são tão separadas como se imaginava, pode-se argumentar que o abismo que as separa é de caráter socialmente construído e isso não significa que ciência e política possuem os mesmos princípios, mas que se reconhece o processo de coprodução que implica aceitar a existência de interferências sociais, econômicas e culturais em ambos os campos.

Portanto, o intuito não é argumentar sobre a não diferenciação entre os campos ou afirmar que ambos operam do mesmo modo. Apenas se reconhece que, na condição de ordenamentos distintos e percebidos pelos atores e redes, eles são dotados de diferenças materiais e que há um jogo constante entre a coprodução e reificação da diferença entre os modos de ordenamento que se expressam nas narrativas dos distintos atores (CARNEIRO; DELATIN; SANDRONI, 2015), por meio de um trabalho de fronteira, expresso em normas, discursos, representações, imagens, leis, instituições etc.

E, com o intuito de interpretar os enquadramentos e os quadros existentes e modificados entre ciência e as políticas públicas, Jasanoff (2004) expõe que o método comparativo pode vir a funcionar melhor quando as entidades a serem comparadas são suficientemente diferentes para apresentar contrastes interessantes, mas suficientemente semelhantes para que as variáveis sejam disciplinadas.

De acordo com a autora, a análise é qualitativa, mas durante o processo de pesquisa, principalmente em análises primárias, técnicas quantitativas podem ser utilizadas no mapeamento de dados e na observância de semelhanças e dissensos entre as unidades comparadas. Essa comparação, no entanto, deve ser edificada em torno do conceito de cultura política e não de categorias estáticas, como atores, interesses ou instituições. 
A cultura política é entendida como os meios sistemáticos pelos quais uma comunidade política faz escolhas coletivas vinculativas. O termo abrange desde os modos de ação institucionalmente sancionados como também códigos não escritos e práticas com as quais a política complementa seus métodos formais, em termos de manutenção da responsabilidade e legitimação na tomada de decisão política (JASANOFF, 2005).

Ou seja, a cultura política em sociedades do conhecimento na contemporaneidade inclui rotinas tácitas, pelas quais o conhecimento coletivo, produzido e validado, abrange formas institucionalizadas de raciocínio e deliberação, além de movimentos pelos quais uma política retira ou coloca questões políticas e científicas.

A análise da cultura política busca capturar estabilidades em práticas sociais e a criação de significados, mas o termo cultura não deve rotular a perspectiva do analista de modo mecânico ou assimétrico (a cultura como marcador do Outro). É necessário sempre realizar um exercício reflexivo sobre como os atores (sejam eles políticos ou cientistas, por exemplo) dão sentido a suas próprias confusões e termos, e, nesse sentido, a cultura deve ser pensada como construída, flexível e sujeita a renovação constante.

Assim, Jasanoff $(2004,2005)$ propõe a realização de estudos de caso comparativo, desde que não efetue prescrições, pois o intuito deve ser o de observar a fabricação dos ordenamentos legítimos. A intenção deve ser o de explorar o conceito de cultura política, com o intuito de interpretar a relação entre conhecimento, tecnologia e poder, em democracias industriais contemporâneas, e expor as relações desses elementos situados em culturas particulares de ação e decisão.

Ou seja, como a cultura política se desenvolve (plays out) em decisões e debates, como essa produção afeta o conhecimento público, como esse conhecimento é avaliado pelo público em geral, composto por uma multiplicidade de identidades e interesses.

Isso significa que uma estratégia comparativa intersectorial (aquilo que se processa em vários setores) - cujo quadro é emoldurado por políticas específicas, que pode, no entanto, ser enquadrado a partir da perspectiva dos atores em suas ações e decisões, além de também estar em contínua mudança, uma vez que se emoldura a própria moldura (quadro - que restringe e ao mesmo tempo configura o olhar) - representa o limite da fronteira, portanto, o descontrole da tentativa de controlar.

As reflexões de Jasanoff se constroem nas perspectivas construtivista e coproducionista da relação entre ciência e política, buscando comparar o enquadramento das questões 
para a tomada de decisões, sua incorporação nas instituições governantes, nos regimes discursivos, e o impacto dessas decisões sobre os atores e suas identidades sociais.

O intuito não é transmitir uma espessura descritiva dos eventos, como realizava Clifford Geertz, ou seguir os objetos, pessoas e trajetórias discursivas, como argumentam Bruno Latour e Marcus, mas capturar regularidades em episódios de mudanças políticas e legitimação democrática.

O foco está nos compromissos normativos autoperpetuantes, que dão à sociedade coerência e solidariedade perante os desafios e as mudanças impostas, ou seja, trata-se de entender como os "imaginários sociotécnicos" - compreendidos como formas imaginadas da vida e da ordem social, que se concentram no desenvolvimento e realização de projetos científicos e/ou tecnológicos inovadores, apesar das divergências culturais "inter-nacionais" e "inter-sectoriais" - permanecem.

Deste modo, pode-se argumentar que o fundamento da pesquisa qualitativa comparada, em Sheila Jasanoff (2005), é o interpretativo e está associado, de certo modo, ao debate da Ciência Política que busca realizar análises comparadas entre países (sendo os Estados unidades primárias de comparação), com o intuito de identificar relações causais independentes e dependentes, a partir de cortes metodológicos, como "mais semelhantes" ou "mais diferentes", em desenhos de pesquisa de small-n ou larger-n, podendo vir a generalizar-se para outras unidades analíticas (TARROW, 1999). Além de se inserir nas reflexões desenvolvidas na área denominada de Estudos Sociais da Ciência e Tecnologia, que focam nos significados, nos discursos, nas epistemes e nas representações, com foco em microescalas (JASANOFF, 2005; GOTTWEIS, 1998).

Baseada nas reflexões de Anderson (1991) e Elman (1997), Jasanoff busca investigar e refletir sobre como as ideias de nacionalidade e de interesse nacional são imaginadas, reimaginadas e representadas em processos de desenvolvimento de políticas tecnocientíficas; portanto, seu método adota uma perspectiva comparativa que se desdobra em dois momentos. Primeiramente, ancorada na ideia de estudos de caso small-n, a autora busca mapear discursos e práticas do que se compreende por imaginários sociotécnicos nacionais (que podem estar relacionados com necessidades nacionais, princípios de solidariedade, temporalidade, competitividade, riscos e benefícios, discursos políticos a respeito de riscos e ética coletiva, juntamente com a ideia de compromisso democrático atrelados ao que ela denomina de cultura política). 
Em um segundo momento (semelhante às análises de estudos de casos múltiplos, mas baseada na perspectiva interpretativa), a autora busca refletir sobre as semelhanças e os dissensos em relação aos casos selecionados, por meio de alguns critérios, como: o debate público, as respostas discerníveis politicamente em nível nacional, os arranjos institucionais, a alteração dos termos existentes do discurso normativo ou a introdução de novos elementos, as novas ou alteradas identidades sociais, dependendo do objeto de reflexão.

Portanto, centrada no que denomina de inter-nacional (cross-national), compreendido como análises que frisam reflexões transversais em distintos níveis (macro, médio e micro), dentro dos Estados, assim como entre os Estados, assim como inter-sectorial (cross-sectorial), que estão em disputas, em movimento, em controvérsias, em diversas agências, organizações, indivíduos, dentre outros, a perspectiva de Jasanoff sobre análise comparativa qualitativa pode vir a ser uma, dentre outras, a colaborar com as discussões metodológicas da Ciência Política na condução de pesquisas, principalmente no que se refere às concepções estáticas e causais de "causas que geram efeitos" ou "efeitos que geram causas".

De fato, perante a complexidade e a proliferação de objetos e intersecções híbridas na atualidade, assim como as tentativas constantes e efetivas de purificação, a Ciência Política pode ganhar com a abordagem de na medida em que essa abordagem ajuda a repensar categorias clássicas da área, como Estado-nação, poder, governo, governança, instituições, opinião pública, razão pública e cultura. Por outro lado, a Ciência Política, justaposta às reflexões de Jasanoff e a autores do campo dos Estudos de Ciência e Tecnologia, pode contribuir com reflexões disciplinares em termos metodológicos, assim como indicar limites interpretativos da análise.

Arrisco-me a afirmar que as contribuições de Sheila Jasanoff podem contribuir com as discussões metodológicas nas análises comparadas qualitativas na Ciência Política, não no sentido de superação (como muitos institucionalistas e neo-institucionalistas expõem ao descreverem a história dos paradigmas históricos na área), mas como outra possibilidade (sem cair no relativismo literal) para as discussões do que se denominou de "culturalista" ou "comportamentalista", frisando não apenas as instituições e os pressupostos causais, mas análises em forte diálogo com a história, com o direito, com as relações 
internacionais, com a antropologia e com a sociologia, como buscam realizar alguns autores do institucionalismo histórico ou do que se denomina de Narrativas Analíticas.

\section{Considerações finais}

Mais do que lógicas explicativas, as análises qualitativas e quantitativas não devem ser interpretadas como perspectivas em oposição, pois representam o jogo constante entre coprodução e reificação da diferença entre modos de ordenamento explicativo sobre o mundo empírico, expressas pela narrativa dos atores, das instituições e suas representações teóricas sobre o mundo.

Metodologicamente, há especificidades entre as duas análises (qualitativa e quantitativa), relacionadas ao objeto de estudo, ao desenho de pesquisa e ao enfoque analítico, o que não significa que uma determinada pesquisa não possa utilizar técnicas ou ferramentas de ambas as tradições. Contudo, é necessário cautela, pois o movimento da procura, assim como da explicação dos dados, deve levar em conta três condições essenciais nas pesquisas qualitativas comparativas: a procura pelas causas que geram um determinado fenômeno, que por sua vez determinam quais variáveis serão dependentes e quais serão independentes, de acordo com o objeto investigado.

Os fenômenos políticos, por mais que possam ser antecipados a partir de estimativas médias, são, por natureza, imprevisíveis e relacionados profundamente ao contexto em que estão inseridos, em termos temporais e espaciais. E a explicação de fenômenos políticos, assim como das agências, das instituições (formais e informais) e dos mecanismos causais, dependendo do enfoque, exerce grande fator explicativo e interpretativo, enquadrando a explicação antes mesmo da coleta de dados.

Se há três condições essenciais da pesquisa qualitativa, uma das características centrais dessas análises é que elas ocorrem sob um número pequeno de casos e com muitas variáveis analíticas, o que, por sua vez, faz que recebam inúmeras críticas. A "nova metodologia qualitativa", no entanto, por meio de um debate crescente, está ofertando respostas coerentes por meio de ferramentas analíticas utilizadas em análises comparadas e estudos de caso.

Nessa tradição qualitativa, por meio de um número pequeno de casos que serão analisados internamente e na coprodução entre o global e o local, Jasanoff afirma que 
a comparação é essencial, mas não deve servir para criar parâmetros ou jogos imitativos entre as unidades de comparação - os Estados-nação -, uma vez que a parametrização comparativa ao longo da história enquadrou unidades estatais, ideias, pessoas, dentre outros, como participantes ou não da política, seja ela local, seja internacional, seja, ainda, global.

A análise comparativa centrada na ideia de cultura política, com base em princípios, tais como identidades, instituições, discursos, representações, dentre outros, pode ser compreendida como uma das análises "novas" dentro dos estudos comparados qualitativos.

Pois, como na metáfora de William Bartes "how can we know the dancer from the dance?", como podemos presumir compreender e explicar resultados em termos de causas distintas e independentes? De acordo com o quadro da coprodução, os objetivos instrumentais do Estado, os conhecimentos e as práticas adotadas para alcançá-los, e os padrões aplicáveis de credibilidade e legitimidade, são construídos em um processo coletivo de ordenamento do mundo. Nesse sentido, precisamos refletir sobre os termos, os pressupostos metodológicos e as teorias empregadas nas análises políticas, a fim de termos suportes com processos de continuidade e mudança, rejeitando, de certo modo, extrema rigidez dos aspectos estruturais nas pesquisas.

\section{Referências}

ANDERSON, B. Imagined communities: reflections on the origin and spread of nationalism. London: Verso, 1991.

BATES, R. H. et al. Analytical narratives. Princeton: Princeton University Press, 1998.

BLYTH, M. Great punctuations: prediction, randomness, and the evolution of comparative political science. The American Political Science Review, v. 100, n. 4, p. 493-498, 2006. http:// dx.doi.org/10.1017/S0003055406062344.

BRADY, H.; COLLIER, D.; SEAWRIGHT, J. Refocusing the discussion of methodology. In: BRADY, H.; COLLIER, D. Rethinking social inquiry: diverse tools, shared standards. Oxford: Rowman \& Littlefield Publishers, 2004. p. 3-21.

CAMPBELL, D. Degrees of freedom and the case study. Comparative Political Studies, v. 8, n. 2, p. 178-193, 1975. http://dx.doi.org/10.1177/001041407500800204.

CARNEIRO, M. J.; DELATIN, D.; SANDRONI, L. Produção e circulação do conhecimento entre cientistas e gestores públicos. In: REUNIÃO DE ANTROPOLOGIA DA CIÊNCIA ETECNOLOGIA (ReACT), 2015, Porto Alegre. Anais... 2015. v. 2, n. 2. 
COLEMAN, J. S. Foundations of social theory. Cambridge: Harvard University Press, 1990.

COLLIER, D. The comparative method. In: FINIFTER, A. (Org.). Political Science: the State of the Discipline II. Washington: American Political Science Association, 1993.

COLLIER, R. B.; COLLIER, D. Shaping the political arena:Critical junctures, the labor movement, and regime dynamics in Latin America. Princeton: Princeton University Press, 1991.

ECKSTEIN, H. Case study and theory in political science. In: GREENSTEIN, F.; POLSBY, N. (Org.). Handbook of Political Science. Reading: Addison, 1975.

ELMAN, M. National imaginations and systems of innovation. In: EDQUIST, C. (Org.). Systems of innovation: technologies, institutions, and organizations. London: Pinter Publishers. 1997. p. 157-173.

FERNANDES, A. S. A. Path dependency e os estudos históricos comparados. BIB, n. 53, p. 79-102, 2002.

FREEDMAN, D. A. On types of scientific enquiry: the role of qualitative reasoning. In: BOXSTEFFENSMEIER, J.; BRADY, H.; COLLIER, D. (Ed.). The Oxford Handbook of Political Methodology. Oxford: Oxford University Press, 2008.

GEDDES, B. How the cases you choose affect the answers you get: selection bias in comparative politics. Political Analysis, v. 2, p. 131-150, 1990. http://dx.doi.org/10.1093/pan/2.1.131.

GEDDES, B. Game theoretic model of reform in latin american democracies. The American Political Science Review, v. 85, n. 2, p. 371-392, 1991. http://dx.doi.org/10.2307/1963165.

GEORGE, A.; BENNETT, A. Case studies and theory development in the social science. Cambridge: MIT Press, 2005.

GEORGE, A.; MCKEOWN, T. Case studies and theories of organizational decision making: advances in informational processing in organizations. Santa Barbara: JAI Press, 1985.

GERRING, J. Case study research: principles and practices. Cambridge: Cambridge University Press, 2007.

GOERTZ, G.; STARR, H. Necessary conditions: theory, methodology, and applications. Lanham: Rowman \& Litllefield, 2002.

GOERTZ, G.; STARR, H. Necessary conditions: theory, methodology and applications. Lanhan: Rowman \& Littlefield, 2003.

GOTTWEIS, H. Governing molecules: the discursive politics of genetic engineering in europe and in the united states. Cambridge: MIT Press, 1998.

JASANOFF, S. (Org.). States of knowledge: the co-production of Science and social order. London: Routledge, 2004.

JASANOFF, S. Designs of nature: science and democracy in Europe and the United States. Princeton: Princeton University Press, 2005.

KING, G.; KEOHANE, R.; VERBA, S. The science of social science: of designing social inquiry. Princeton: Princeton University Press, 1994. 
LAW, J. Organizing modernity. Oxford: Blackwell, 1994.

LEVI, M. A model, a method, and a map: rational choice in comparative and historical analysis. IN: LICHBACH, M.; ZUCKERMAN, A. (Org.). Comparative politics: rationality, culture and structure. Cambridge: Cambridge University Press, 1997. p. 19-41.

LEVI, M. An analytical narrative approach to puzzles and problems. In: SHAPIRO, I.; SMITH, R. M.; MASOUD, T. E. (Org.). Problems and methods in the study politics. Cambridge: Cambridge University Press, 2004.

LIJPHART, A. Comparative politics and the comparative method. The American Political Science Review, v. 65, n. 03, p. 682-693, 1971. http://dx.doi.org/10.2307/1955513.

MAHONEY, J. After KKV: the new methodology of qualitative research. World Politics, v. 62, n. 1, p. 120-147, 2010. http://dx.doi.org/10.1017/S0043887109990220.

MAHONEY, J.; GOERTZ, G. A tale of two cultures: contrasting quantitative and qualitative research. Political Analysis, v. 14, n. 3, p. 227-249, 2006. http://dx.doi.org/10.1093/pan/mpj017.

MAHONEY, J.; GOERTZ, G. The possibility principle: choosing negative cases in comparative research. The American Political Science Review, v. 98, n. 4, p. 653-669, 2004. http://dx.doi. org/10.1017/S0003055404041401.

MAHONEY, J.;TERRIE, P. L. Comparative-historical analysis in contemporary political science. In: BOX-STEFFENSMEIER, J. M.; BRADY, H. F.; COLLIER, D. (Org.). The Oxford Handbook of political methodology. New York: Oxford University Press, 2008. p. 737-754.

MARQUES, I. C. et al. Sheila Jasanoff: localizando o global. Historia, Ciências, Saúde: Manguinhos, v. 198, n. 3, p. 993-1039, 2012.

MAZZOCCHI, F. Western science and traditional knowledge. EMBO Reports, v. 7, n. 5, p. 463-466, 2006. PMid:16670675. http://dx.doi.org/10.1038/sj.embor.7400693.

MCKEOWN, T. Case studies and the statistical world view. International Organization, v. 53, n. 1, p. 161-190, 1994. http://dx.doi.org/10.1162/002081899550841.

MERTON, R. K. The bearing of empirical research on sociological theory. In: MERTON, R. K. On theoretical sociology: five essays, old and new. New York: Free Press, 1967, p. 156-171.

PASQUARELLI, B. V. L. Política comparada: tradições, métodos e estudos de caso. Agenda Política: Revista de Discentes de Ciência Política da UFSCar, v. 2, n. 2, p. 10-38, 2014.

PLOTT, C. R. Will economics become na experimental science? Southern Economic Journal, v. 57, p. 901-920, 1991.

PRZEWORSKI, A.; TEUNE, H. The logic of comparative social inquiry. New York: John Wiley, 1970.

PUTNAM, R. D. Making democracy work: civic traditions in modern Italy. New Jersey: Princeton University Press, 1993.

RAGIN, C. The comparative method: moving beyond qualitative and quantitative strategies. Berkeley: University of California Press, 1987. 
REZENDE, F. C. A nova metodologia qualitativa e as condições essenciais de demarcação entre desenhos de pesquisa na Ciência Política Comparada. Revista Política Hoje, v. 20, n. 1, p. 218-252, 2011a.

REZENDE, F. C. Razões emergentes para a validade dos estudos de caso na ciência política comparada. Revista Brasileira de Ciência Política, n. 6, p. 297-337, 2011 b.

RORTY, R. Phony science wars. Atlantic Monthly, v. 284, n. 5, p. 120-122, 1999.

SAID, E. W. Orientalism. London: Routledge \& Kegan Paul, 1978.

TARROW, S. Expanding paired comparison: a modest proposal. APSA-CPNewsletter, v. 10, n. 2, p. 9-12, 1999.

THELEN, K. Historical institutionalism in comparative politics. Annual Review of Political Science, v. 2, n. 1, p. 369-404, 1999. http://dx.doi.org/10.1146/annurev.polisci.2.1.369.

TROCHIM, W. Outcome Pattern Matching and program theory. Evaluation and Program Planning, v. 12, n. 4, p. 355-366, 1989. http://dx.doi.org/10.1016/0149-7189(89)90052-9.

YANOW, D.; SCHWARTZ-SHEA, P.; FREITAS, M. J. Case study research in political science. In: MILLS, A. J.; DUREPOS, G.; WIEBE, E. (Ed.). Encylopedia of case study research. Los Angeles: Sage, 2008.

Recebido: 02 dez., 2016 Aceito: 26 mar., 2017 